\title{
Effect of Coupling Types on Synchronization of Weakly Coupled Bursting Neurons
}

\author{
Meili Lu ${ }^{1}$, Bei Liu², Yanqiu Che ${ }^{2}$, a Chunxiao $\operatorname{Han}^{2}$ \\ ${ }^{1}$ School of Information Technology and Engineering, 2Tianjin Key \\ Laboratory of Information Sensing \& Intelligent Control, Tianjin \\ University of Technology and Education, Tianjin, 300222, China \\ ayqche@tju.edu.cn
}

\begin{abstract}
Weak coupling connections are ubiquitous in neuronal system. For neurons with both spiking and bursting dynamics, bursting synchronization, which has been found to be related with a number of abnormal brain rhythms, is easier to occur at smaller synaptic strengths. In this paper, we examine the effect of the synaptic types on the synchronization properties of the weakly coupled bursting neuronal oscillators by means of phase-model reduction. The bifurcation analysis clarifies how the synaptic types affect the existence and stability of in-phase, anti-phase and out of phase synchronization states. The results show that electrical coupling and inhibitory coupling neurons have stable anti-phase synchronous solutions, while excitatory coupling neurons exhibit out of phase synchronous behaviors.

Keywords: Bursting Neurons; Synchronization; Weak Coupling; Phase Response Curve.

\section{Introduction}

Synchronization in neuronal system is a significant problem in computational neuroscience, which has received a great deal of attention in the past decades [1]. Experimental evidence demonstrates that synchronized neuronal activity has been suggested as particularly relevant in neural encoding and information process [2]. Some neurons in the brain display bursting behavior. As a unit of neural information, it contains two time scales with fast spiking and relative slow periodic oscillations. Bursting can increase the reliability of communication between neurons [3, 4]. Bursting synchronization can be influenced by many factors, such as coupling strength and synaptic coupling types [5-11]. Two types of synaptic connection, electrical (or gap junction) and chemical coupling, are quite different. Several studies have revealed the effects of different couplings on the sychronization of spiking neurons [6-11], however, the case for bursting neurons has not been fully understood. Phase model method, which captures how timing of one neuron affects the other one on a long time scale, provides a powerful tool to study network behaviors of weakly coupled neurons, especially for synchronization [9-12].
\end{abstract}


In this paper, we investigate the effect of synaptic types on synchronous behaviors of weakly coupled bursting neurons via phase model reduction method. We firstly briefly describe the phase-model reduction method. Then we introduce the bursting neuronal model developed from the Morris-Lacor model [13] as well as their couplings. Futher, the membrane potential time-response and corresponding phase response curves (PRC) are explored. Then, we analyze the electrical and chemical coupling connections and the role of the type of coupling. Finally, a summary is also given.

\section{Phase-Model Reduction Method}

Consider a oscillation system $\dot{x}=f(x)$ with a stable limit cycle $\Gamma_{0}(t)$ disturbed by an external input $\epsilon p(t)$ with $\epsilon \square 1$, the dynamics $\dot{x}=f(x)+\epsilon p(x, t)$ can be reduced to the following phase system by introducing phase variable $\theta(t)=t+\varphi$ with $t$ capturing fast free-running oscillation $\dot{x}=f(x)$, and $\varphi$ capturing slow network-induced phase deviation from the natural oscillation.

$$
\dot{\theta}=\frac{\partial \theta}{\partial x} \cdot \dot{x}=\frac{\partial \theta}{\partial x} \cdot(f(x)+\varepsilon p(x, t))=1+\varepsilon \frac{\partial \theta}{\partial x} \cdot p(x, t)
$$

The Phase Response Curve (PRC) is defined as $Q(\theta)=\left.(\partial \theta / \partial x)\right|_{\Gamma_{0}(\theta)}$, then we have $\dot{\varphi}=\varepsilon Q(t+\varphi) p(x(t+\varphi), t)$.

For two identified coupled neurons considered in our case, the phase system can be expressed as:

$$
\dot{\varphi}_{i}=\varepsilon Q_{i}\left(t+\varphi_{i}\right) \cdot p_{i j}\left(x_{i}\left(t+\varphi_{i}\right), x_{j}\left(t+\varphi_{j}\right)\right), \quad i, j=1,2
$$

Since the phase deviations $\varphi_{i}$ are much slower than the oscillations (variable $t$ ), system (2) can be transformed into the following form by means of the method of averaging, $\dot{\varphi}_{i}=\varepsilon H_{i j}\left(\varphi_{j}-\varphi_{i}\right)$, where $i, j=1,2$ and $H_{i j}\left(\varphi_{j}-\varphi_{i}\right)=(1 / T) \int_{0}^{T} Q(t) \cdot p_{i j}\left(\Gamma_{0}(t), \Gamma_{0}\left(t+\varphi_{j}-\varphi_{i}\right)\right) d t$ is the effective coupling function.

Let $\eta=\varphi_{2}-\varphi_{1}$ denote the phase difference between the oscillators, then the above system becomes $\dot{\eta}=\varepsilon G(\eta)$, where $G(\eta)=H_{21}(-\eta)-H_{12}(\eta)$ is the anti-symmetric part of the coupling. All equilibriums are solutions to $G(\eta)=0$, and they are intersections of the horizontal zero line with the graph of $G(\eta)$. 
They are stable if the slope of the graph is negative at the intersection. Since the For identical oscillators, $H(\eta)$ is an odd function (i.e., $H(-\eta)=-H(\eta)$ ), $\eta=0$ and $\eta=T / 2$ are always equilibriums, corresponding to the in-phase and anti-phase synchronized solutions.

\section{Bursting Neuronal Model}

By introducing a slow potassium channel to the traditional Morris-Lecar neuronal model, one obtains a bursting neuronal model consisting of three differential equations [11]

$$
C_{\mathrm{M}} \dot{V}=I_{\text {ext }}-I_{\text {ion }}, \quad \dot{N}=\phi\left(N_{\infty}-N\right) / \tau_{N}, \quad \dot{S}=\delta\left(S_{\infty}(V)-S\right) / \tau_{S}(V) .
$$

where

$I_{\text {ion }}=\bar{g}_{\mathrm{Ca}} M_{\infty}\left(V-V_{\mathrm{Ca}}\right)+\bar{g}_{\mathrm{K}} N\left(V-V_{\mathrm{K}}\right)+g_{\mathrm{L}}\left(V-V_{\mathrm{L}}\right)-\bar{g}_{\mathrm{KS}} S\left(V-V_{\mathrm{K}}\right)$, $V$ represents membrane potential, $N \in[0,1]$ represents the potassium activation variable, $S$ is the gate variable controlled by slow potassium ions. $C_{\mathrm{M}}$ is the membrane capacitance, $I_{\text {ext }}$ is the externally-applied DC current. The parameters $V_{\mathrm{Ca}}, V_{\mathrm{K}}$ and $V_{\mathrm{L}}$ represent the equilibrium potentials of calcium, potassium and leak current, respectively. $\bar{g}_{\mathrm{Ca}}, \bar{g}_{\mathrm{K}}, \bar{g}_{\mathrm{KS}}$ and $g_{\mathrm{L}}$ are the maximal conductance of the corresponding ionic currents. $M_{\infty}, N_{\infty}$ and $S_{\infty}$ are nonlinear functions of $V$, given by $M_{\infty}=0.5\left[1+\tanh \left(\left(V-V_{1}\right) / V_{2}\right)\right]$, $N_{\infty}=0.5\left[1+\tanh \left(\left(V-V_{3}\right) / V_{4}\right)\right]$ and $S_{\infty}=0.5\left(1+\tanh \left(\left(V-V_{5}\right) / V_{6}\right)\right)$, respectively, where $V_{1}, V_{3}$ and $V_{5}$ are the activation midpoint potential at which the corresponding currents are half activated, $V_{2}, V_{4}$ and $V_{6}$ denote the slope factor of the activation. The time constants $\tau_{N}$ and $\tau_{S}$ about the potassium activation are described by $\tau_{N}=1 / \cosh \left(\left(V-V_{3}\right) / 2 V_{4}\right)$ and $\tau_{S}=1 / \cosh \left(\left(V-V_{5}\right) /\left(2 V_{6}\right)\right)$.

Throughout this paper, all the parameters involved in ML model are fixed as values $C_{\mathrm{M}}=20 \mu \mathrm{F} / \mathrm{cm}^{2}, \quad \bar{g}_{\mathrm{Ca}}=4 \mathrm{mS} / \mathrm{cm}^{2}, \bar{g}_{\mathrm{K}}=8 \mathrm{mS} / \mathrm{cm}^{2}$, $g_{\mathrm{L}}=2 \mathrm{mS} / \mathrm{cm}^{2}, \bar{g}_{\mathrm{KS}}=0.5 \mathrm{mS} / \mathrm{cm}^{2}, V_{\mathrm{Ca}}=120 \mathrm{mV}, V_{\mathrm{K}}=-80 \mathrm{mV}$, $V_{\mathrm{L}}=-60 \mathrm{mV}, V_{1}=-1.2 \mathrm{mV}, V_{2}=18 \mathrm{mV}, V_{3}=12 \mathrm{mV}, V_{4}=17.4 \mathrm{mV}$, 
$V_{5}=-10 \mathrm{mV}, V_{6}=4 \mathrm{mV}, \phi=0.22 \mathrm{~ms}^{-1}, \delta=0.001 \mathrm{~ms}^{-1}, \quad$ and $I_{\mathrm{ext}}=42 \mathrm{pA} / \mathrm{cm}^{2}$.

The dynamics of the two electrically coupled system is described as:

$$
\dot{V}_{i}=\frac{1}{C_{\mathrm{m}}}\left[I_{\mathrm{ext}}^{i}-I_{\mathrm{ion}}^{i}-g_{\mathrm{c}}\left(V_{j}-V_{i}\right)\right], \quad \dot{N}_{i}=\phi \frac{N_{\infty}\left(V_{i}\right)-V_{i}}{\tau_{N}\left(V_{i}\right)}, \dot{S}_{i}=\delta \frac{S_{\infty}\left(V_{i}\right)-S_{i}}{\tau_{S}\left(V_{i}\right)}
$$

$$
\text { where } \quad g_{c} \quad \text { is coupling strength, }
$$$$
I_{\text {ion }}^{i}=\bar{g}_{\mathrm{Ca}} M_{\infty}^{i}\left(V_{i}-V_{\mathrm{Ca}}\right)+\bar{g}_{\mathrm{K}} N_{i}\left(V_{i}-V_{\mathrm{K}}\right)+g_{\mathrm{L}}\left(V_{i}-V_{\mathrm{L}}\right)+\bar{g}_{\mathrm{KS}} S_{i}\left(V_{i}-V_{\mathrm{K}}\right)
$$

The coupling function is $p_{i j}\left(x_{i}, x_{j}\right)=\left(-\left(V_{j}-V_{i}\right) / C_{M}, 0\right)$. Then the reduced phase model is $\dot{\eta}=g_{c} G(\eta)=g_{c}(H(-\eta)-H(\eta))$ with $H(\eta)=-1 /\left(C_{m} T\right) \int_{0}^{T}\left(Q_{1}(t) \cdot\left(V_{0}(t+\eta)-V_{0}(t)\right)\right) d t$, where $V_{0}(t)$ is the membrane potential on the limit cycle, and $Q_{1}(t)$ is the corresponding PRC.

The dynamics of the two chemically coupled ML systems is described as:

$\dot{V}_{i}=\frac{1}{C_{\mathrm{m}}}\left[I_{\text {ext }}^{i}-I_{\text {ion }}^{i}-g_{\text {syn }} S_{j}\left(V_{i}-V_{\text {syn }}^{\text {post }}\right)\right], \dot{N}_{i}=\phi \frac{N_{\infty}\left(V_{i}\right)-V_{i}}{\tau_{N}\left(V_{i}\right)}, \dot{S}_{i}=\delta \frac{S_{\infty}\left(V_{i}\right)-S_{i}}{\tau_{s}\left(V_{i}\right)}, \dot{s}_{i}=\alpha s_{\infty}\left(V_{i}\right)\left(1-s_{i}\right)-\beta s_{i}$

where $s_{\infty}(V)=1 /\left(1+\exp \left(-\left(V-V_{\text {syn }}^{\text {pre }}\right) / \sigma\right)\right), g_{\text {syn }}$ is the conduction of the synaptic channel, $V_{\text {syn }}^{\text {post }}$ and $V_{\text {syn }}^{\text {pre }}$ are the post-synaptic and pre-synaptic threshold potential, respectively, $s$ is the rate of connecting receptors, $\alpha$ and $\beta$ are time constants. The excitatory coupling parameters are $V_{\text {syn }}^{\text {post }}=0 \mathrm{mV}$, $V_{\text {syn }}^{\text {pre }}=2 \mathrm{mV}, \alpha=2.2, \beta=0.19, \sigma=4.5$. Inhibitory coupling parameters are $V_{\text {syn }}^{\text {post }}=-70 \mathrm{mV}, \alpha=10, \beta=0.18$. The coupling strength used is $g_{\text {syn }}=0.05 \mathrm{mS} / \mathrm{cm}^{2}$.

The coupling function can be described as $p_{i j}\left(x_{i}, x_{j}\right)=\left(-s_{j}\left(V_{i}-V_{\text {syn }}^{\text {post }}\right) / C_{M}, 0,0\right)$. Then the reduced phase model is $\quad \dot{\eta}=g_{\text {syn }} G(\eta)=g_{\text {syn }}(H(-\eta)-H(\eta)) \quad$ with $H(\eta)=-1 /\left(C_{m} T\right) \int_{0}^{T}\left(Q_{1}(t) s_{0}(t+\eta) \cdot\left(V_{0}(t)-V_{\text {syn }}^{\text {post }}\right)\right) d t$ where $V_{0}(t)$ is the membrane potential on the limit cycle of the free-running oscillation as shown in 
Fig. 1 (a). $s_{0}(t)$ is the value of $s$ corresponding to $V_{0}(t)$, and $Q_{1}(t)$ is the corresponding PRC as shown in Fig. 1 (b).
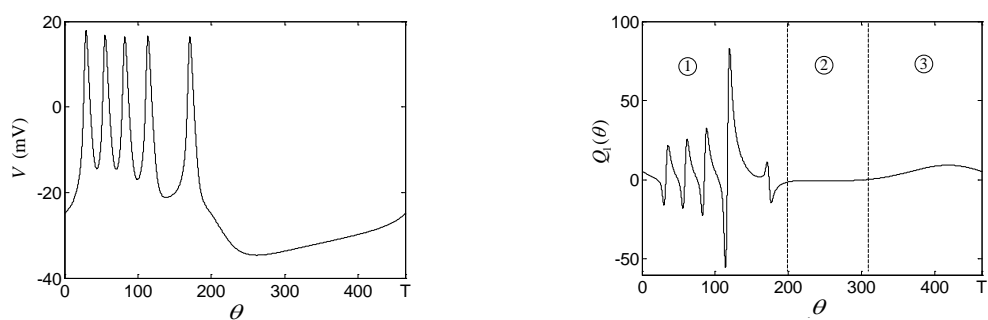

Figure 1: (a) The membrane potential on the limit cycle of the free-running oscillation; (b) The corresponding PRC

\section{Results and Analysis}

Average coupling function and phase-difference coupling function in the electrically coupled system are respectively shown in Figure 2 (a) and (b). And the response of the coupling system in different initial phase conditions is shown in Figure 2 (c), which is consistent to the theoretical results. The system can show many stable synchronous states including in-phase and anti-phase synchronization. Among these attractors, the anti-phase synchronization has the largest attractive domain and the strongest robustness to parameters and input perturbations.

The responses of excitatory and inhibitory synaptic coupled systems are respectively shown in Figure 3 and Figure 4. Coupled bursting neurons with excitatory synapses behave as bi-stable out of phase synchronization, while the in-phase and anti-phase synchronous solutions are both unstable. The system in inhibitory synapses has multiple steady-state and the anti-phase synchronous solution is stable in a wide range, similar to the case with electrical coupling. Compared with electrical coupling, amplitudes of average coupling function and phase-difference coupling function in chemical coupling are much larger, so the synchronous solution converges much faster.

\section{Conclusions}

In this paper, the synchronization of weakly coupled bursting neuronal oscillators in different types of coupling is analyzed. The dynamics of the phase difference between the oscillators are well described in the scheme of phase-model reduction. The roles of different synapses are examined extensively. The fixed points corresponding to the various phase-locked states are identified. The shape of the effective coupling function determines the existence and the stability of those fixed points. The analysis clarifies how the type of coupling affects the existence and stability of in-phase, anti-phase and out of phase synchronization states. Electrical coupling and inhibitory coupling neurons have stable anti-phase 
synchronous solutions but excitatory coupling neurons have out of phase synchronous solutions.
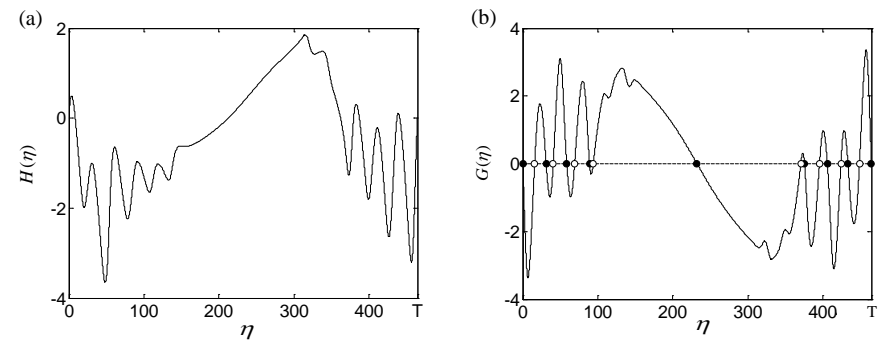

(c)

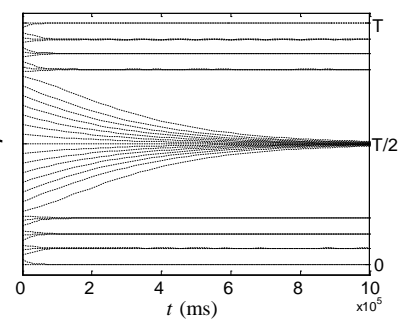

Figure 2: The phase response of excitatory coupled bursting neurons.
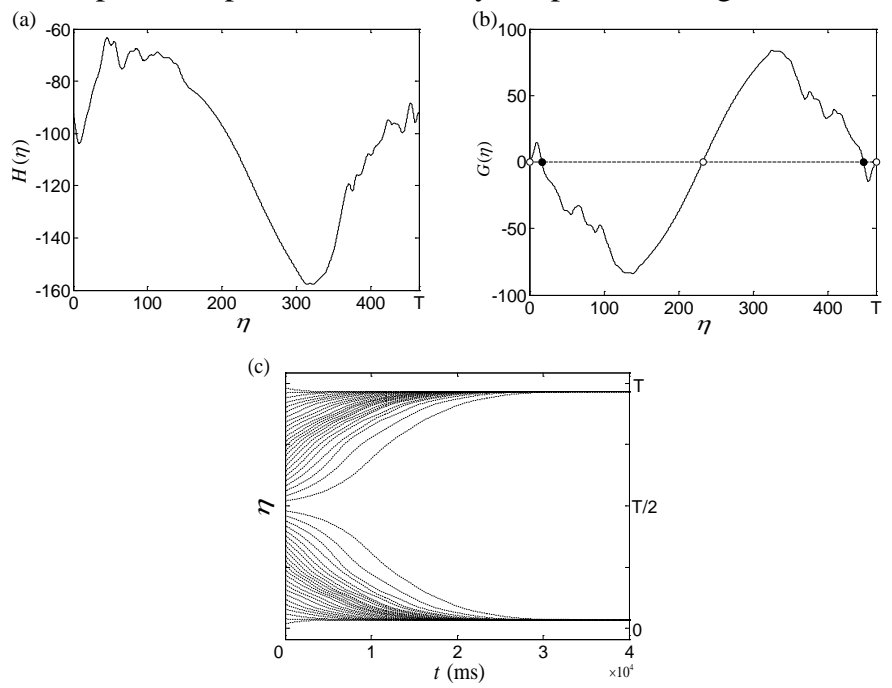

Figure 3: The phase response of excitatory coupled bursting neurons. 

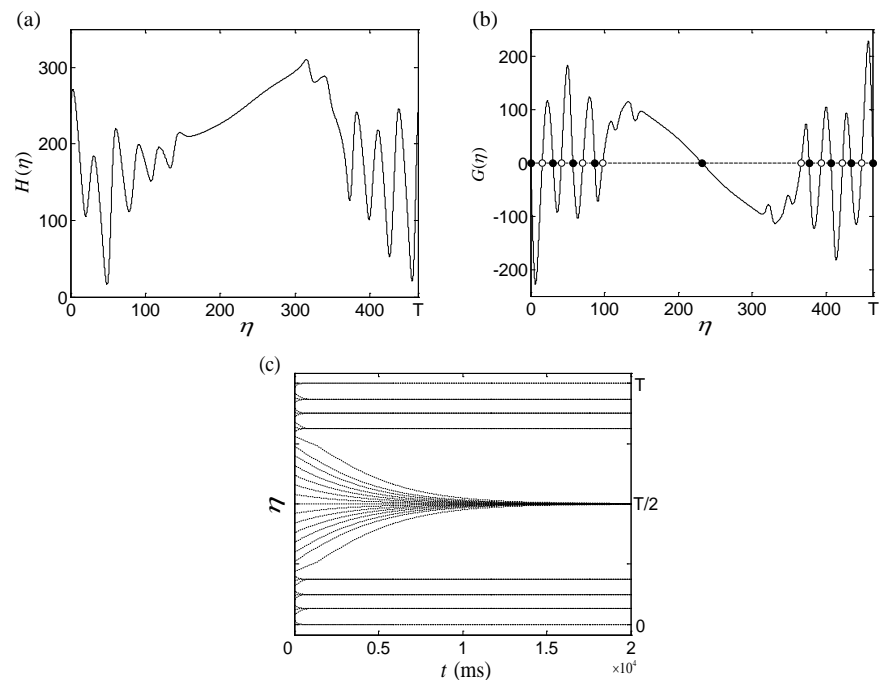

Figure 4: The phase response of inhibitory coupled bursting neurons.

\section{Acknowledgments}

This work is supported by NSFC under Grants No. 50907044, No. 60901035, No. 61072012, No. 61172009, and No. 61104032 and the No. 20100819). We would also acknowledge the support of Tianjin University of Technology and Education (Grants No. KJY1305, and No. KJ11-04

\section{References}

[1] J. Kurths, A. Pikovsky and M. Rosenblum, Synchronization: a universal concept in nonlinear science (University Press, Cambridge: Cambridge, 2001)

[2] Uhlhaas PJ, Singer W: Neuron. Vol. 52(2006), p. 155.

[3] Lisman JE: Trends in neurosciences. Vol. 20(1997), p. 38-43.

[4] Buzsaki G, Rhythms of the Brain (University Press, Oxford: Oxford, 2006)

[5] Belykh I, de Lange E, Hasler M: Physical review letters. Vol. 94(2005).

[6] Chow CC, Kopell N: Neural Computation. Vol. 12(2000), p. 1643.

[7] Lewis TJ, Rinzel J: J Comput Neurosci. Vol. 14(2003), p. 283.

[8] Park E H, Barreto E, Gluckman B J, et al.: J Comput Neurosci, Vol. 19(2005), p. 53. 
[9] Che Y, Zhang S, Wang J, Han C, Wei X, Deng B, Intelligent Control and Information Processing (ICICIP). Vol. 2(2011), p. 1140.

[10] Hao P, Li R, Che Y, Han C, Chinese Control and Decision Conference (CCDC). (2013), p. 2076.

[11] Li R, Che Y, Hao P, Li X, Applied Mechanics and Materials. Vol. 519-520 (2014), p. 846.

[12] Hoppensteadt FC, Izhikevich EM, Weakly connected neural networks (Springer, New York, 1997)

[13] Morris C, Lecar H: Biophysical journal, Vol. 35(1981), p. 193. 